\title{
The Cardio Kinesiograph System
}

\author{
Mohammad Reza Dawoudi* \\ Turku University of Applied Sciences, Finland
}

Submission: August 17, 2017; Published: August 29, 2017

*Corresponding author: Mohammad Reza Dawoudi, Turku University of Applied Sciences, Finland, Email: m.reza.dawoudi@abo.fi

Abstract

The Cardio Kinesiograph System (CKinG) is a novel computerized diagnostic system incorporating a computer model of cardiac kinesis. Cardiac Kinesis (CK) is the interpretation of heart movement or electrical activity of specialized cardiac muscle cells in response to biochemical reactions. The mechanical events occurring during the cardiac cycle consist of changes in pressure in the ventricular chamber which cause(s) blood to move in and out of the ventricle [1]. The events of the cardiac cycle start with an electrical signal and proceed through excitationcontraction coupling (which involves chemical and mechanical events) to contraction of the ventricle (pressure generation) and ejection of blood (flow) into the pulmonary and systemic circulations [2]. Thus, we can characterize the cardiac cycle by tracking changes in ventricular volume (LVV), ventricular pressure (LVP), left atrial pressure (LAP) and aortic pressure (AoP) [3]. In the other hand, because electrical events always precede mechanical events in the cardiac cycle, distortions of a part or parts of the electrical signal have been used as diagnostic indicators of both electrical and mechanical dysfunctions of the heart muscle [4]. There are numerous methods and technical systems available for diagnosis or predict heart disease. However, medical errors and undesirable results [5] in these systems are reasons for a need for conventional computer-based diagnosis of heart diseases systems. The purpose of this study is to design and develop a system that can observe and analyze Kinesiographical Cardiac basted on statistical models and identifies some fundamental characteristic of heart motions.

Keywords: Cardiovascular biomechanics; Statistical analysis methods; Medical heart imaging modalities; DNA modelization in biomedical image

\section{Introduction}

Since 1950 cardiologists have studied the functions of heart moments to employ them in the diagnostics of ischemic heart disease (IHD). Indeed, changes of the movements have found their diagnostic application in this field. If blood supply to a certain area of ventricular myocardium is insufficient the contractions in this area diminish and even ceases. After systolic increase in ventricular pressure this area dilates and forces intercostal tissues out, causing a "bulge" wave on the record [6]. Cardiokymography was one of several noninvasive techniques able to detect coronary artery disease. It can qualitatively determine abnormal left ventricular motion, and, based on animal models, this can be directly related to abnormalities in the left coronary artery [7]. Nevertheless sensitivity of cardiokymography in detecting patients with ischaemic left ventricular wall motion abnormalities depended on the extent of left ventricular ischaemia [8]. Cardiokymography is no longer valid [9]. Today Computer Aided Diagnosis (CAD) is one of the trusted methods in the field of medicine [10]. Advances in medical imaging and image processing techniques have greatly enhanced interpretation of medical images. Computer aided diagnosis (CAD) systems based on these techniques play a vital role [11] in the early detection of cardiovascular diseases and hence reduce death rate. CAD is the most preferable method for the initial diagnosis of heart disease. The combination of Digital and Medical Image Processing, Cardiac Electrophysiology, Ventricular Pressure-Volume Technique and Phonocardiogram etc makes the CAD system more reliable and efficient. One example of the medical applications in computer aided diagnosis is the detection system for heart disease based on Cardiovascular Magnetic Resonance (CMR). Cardiovascular magnetic resonance of the heart provides a potentially useful way to assess cardiac mechanical function. Besides CMR, positron emission tomography (PET), and cardiac CT are able to illustrate kinesis of cardiac muscles. Perfusion imaging with cardiac PET is used clinically to produce images of myocardial blood flow, aiding the diagnosis of coronary artery disease and the monitoring of condition of coronary circulation in response to treatment. [12] Clinical imaging in positron emission tomography (PET) is often performed using single-time-point estimates of tracer uptake or static imaging that provides a spatial map of regional tracer concentration [13]. However, dynamic cardiac technics (e.g. PET, Myocardial perfusion imaging and Dynamic cardiac SPECT [14] are used to estimate rate parameters activity of myocardial blood flow, and there are limited studies evaluating the role of Cardiovascular Magnetic Resonance and cardiac PET and cardiac CT for the assessment of cardiac kinesis. Therefore, scientific 
communities in computational cardiovascular science [15] have contributed to developing mathematical models and algorithms to improve efficiency of cardiac safety data management in clinical trials. In this way in 2014 Narjes B. A. and et al proposed a new method based on a mathematical model, "Fourier Transform" which calculates an amplitude parametric image for the assessment of cardiac kinetics. This image, calculated from the Cine MR images, allows the localization and quantification of abnormalities related to difference in contraction and their extent [16]. In 2015 Zakynthinaki has also presented effective mathematical model of heart rate kinetics in response to movement. She made conclusion that the new model is able not only to provide important information regarding an individual's cardiovascular condition but to also simulate and predict heart rate kinetics for any given exercise intensities [17]. The existing models of cardiac kinetics focus mainly on amplitude images or are limited to simulation of biological transformation. The present study provides a novel mathematical model of cardiac kinesis based on visual presentation of numerical data (obtained through the cardiac kinesis) in the form of graph, with a particular focus on Human Cardio Kinesiograph Analysis. The purpose of this study was to design, develop and evaluate a novel method for Kinesiograph of Cardiac for the clinical assessment of cardiac and vascular function.

\section{Methods and Materials}

The system includes the following steps:

\section{Data collection}

In order to obtain an accurate data, the Cardiovascular Magnetic Resonance imaging (CMR), Cardiovascular Ultrasound or Cardiac Computed Tomography are produced better performances for detection of cardiac mobility. However, CMR is provided the most comprehensive anatomic picture for patient selection [18]. The system therefore is obtained relevant information from the CMR.

\section{Video quality assessment}

Different medical imaging methods may introduce common artifacts include image distortion, signal pileup (bright regions), and image dropout (area without signal), [19] therefore the quality assessment is an important factor at the operational level. The assessment of quality of video depends upon the type of distortion [20]. Numerous video quality assessment methods and metrics have been proposed over the past years with varying computational complexity and accuracy [21]. We utilize different forms of quality assessment methods, however the merit of these methods is often judged by assessing the quality of a set of results through lengthy user studies [22].

\section{Motion estimation and inertial measurements}

Motion estimation is the process of determining the movement of blocks between adjacent video frames (MathWorks). Efficient and accurate motion estimation is an essential component in the domains of image sequence analysis [23] and medical video processing. The estimation of motion is also important from the viewpoint of matching metric technique, which is computed the context similarity between two images. There exist several methods for motion estimation image, and video processing (e.g. pixel-based motion estimation, block-based motion estimation, optical flow method) [24]. In this study in order to increase the computational accuracy and improve efficiency in solving problem, DNA Modeling method in biomedical image matching has been proposed. The method is based on the linear mapping and the one-to-one correspondences between point features extracted from the frames and on calculating similarities in pixel values. This correspondence is determined by comparing two strings constructed from pixel values of the frames. The method uses a table called the Quarter Code table, which is the set of characters and numbers. In this table every number between 0 and 255 is translated into a unique string of four letter alphabet. Letters A, C, G, T are chosen, since they are the same as used in DNA sequences. In this way it possible to utilize tools originally programmed to DNA sequences analysis. When all pixel values of the frames (images) are converted to virtual DNA sequences, one can show the differences between two virtual DNA sequences.

\section{Visual representation of numerical data in the form of graphs}

The E-value gives a measure of the similarity of sequences. From this function we can obtain the correlation coefficient which will give us a single value of similarity. The rate of similarity between sequences (frames) is plotted as a graph and it's appearing in the Monitor.

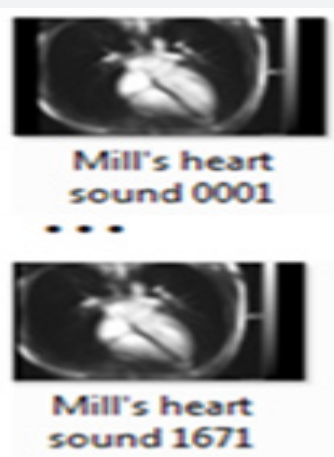

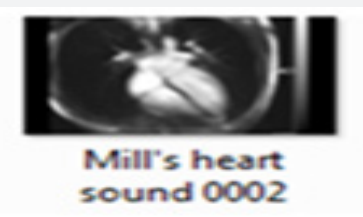

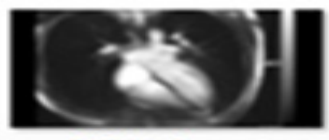

Mill's heart sound 0003

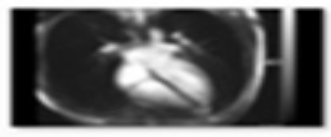

Mill's heart sound 1672

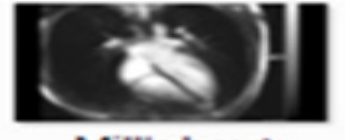

Mill's heart sound 1673
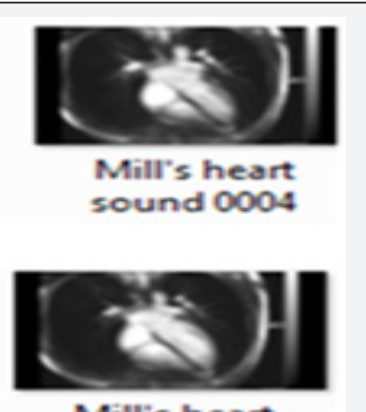

Mill's heart sound 1674

Figure 1: Image extraction from heart MRI video with mill's sound 1 sample. 


\section{Experiment Results}

We demonstrate the system by performing experiment 2D cardiac CMR video (Source: HBSNS library). The practical framework consists of four steps (Figure 1):

\section{Step 1: Extract Frames from cardiac MRI Video}

The information is obtained by extracting frames from CMR imaging video. There are different tools in order to extract frames from cardiac MRI Video (e.g. Free Video to JPG Converter, VLC or VirtualDud).

\section{Step 2: Adjacent Frames Comparisons}

The difference between two adjacent frames is used to estimate motion direction and magnitude. This process has been implemented within a tool called Image Diff. The Perforce image diff tool enables researchers to compare two adjacent frames. The following represents pixels difference value (Percent Changed; Pixels) and color difference value (Percent Change; Color) between adjacent Frames (Figure 2).

\begin{tabular}{|c|c|c|c|}
\hline Frames & $\begin{array}{l}\text { Percent Changed } \\
\text { (Pixe1s) }\end{array}$ & $\begin{array}{l}\text { ercent Changed } \\
\text { (Color) }\end{array}$ & reshold \\
\hline $18 \quad 19$ & 8.13 (start point) & ) 1.08 (start point) & 49 \\
\hline 1920 & 2.09 & 0.22 & 49 \\
\hline$\cdots$ & & & \\
\hline 113114 & 1.31 & 0.13 & 49 \\
\hline
\end{tabular}

Step 3: Presenting Data in Graphic Form

In the final step the percentage of change (pixels and color) from one value to another, between frames are plotted as a graph (Figure $3 \& 4$ ).

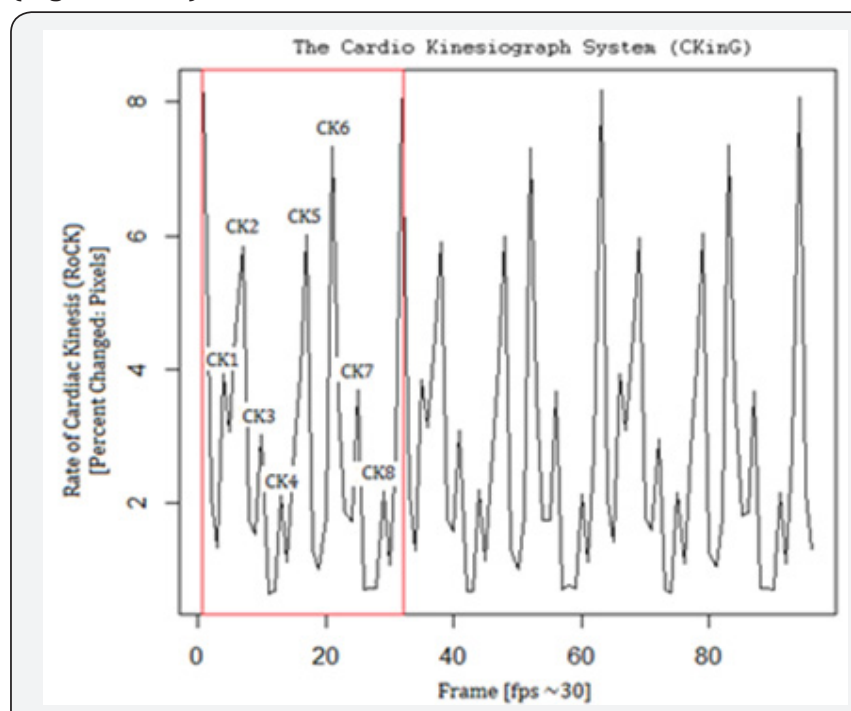

Figure 3: Measured cardiac kinesiography signal three cardiac cycle, there are eight CK (cardiac kinese) events (CK1, CK2, CK3, CK4, CK5, CK6, CK7, CK8, CK9) for each cycle. There are labeled for the cycle.

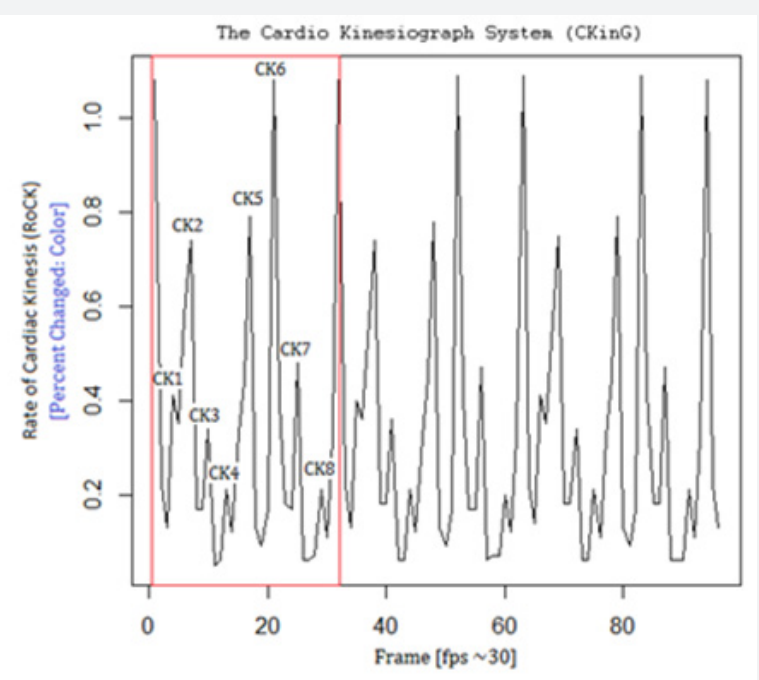

Figure 4: Measured cardiac kinesiography signal three cardiac cycle, there are eight CK (cardiac kinese) events (CK1, CK2, CK3, CK4, CK5, CK6, CK7, CK8, CK9) for each cycle. There are labeled for the cycle.

\section{References}

1. Burkhoff D (2002) Mechanical properties of the heart and its interaction with the vascular system. Cardiac Physiology, pp. 23.

2. Arthur B, Vikki H, Antonio V, Alfred N (2011) Biomedical Engineering Principles. CRC Press Taylor \& Francis Group, India.

3. Sameh G, Adel M, Ali A (2013) Innovative Artificial Neural Networks Based Decision Support System for Heart Diseases Diagnosis. Journal of Intelligent Learning Systems and Applications 5(3).

4. Khaiutin VM, Lukoshkova EV, Sheroziia GG (2004) Computer cardiokymography. On its way to long-term noninvasive monitoring of cardiac performance in daly life. Ross Fiziol Zh Im I M Sechenova 90(5): 609-624.

5. (1987) Diagnostic and therapeutic technology assessment. Intrauterine devices. JAMA 257(21): 2973-2974.

6. Scherhag AW, Pfleger S, Ceconi C, Voelker W, Gehring J, et al. (1997) Evaluation of signal-averaged cardiokymography for the detection of ischaemic left ventricular dysfunction. Int J Cardiol 59(3): 305-312.

7. Clifford Goodman. (1988). Medical Technology Assessment Directory: A Pilot Reference to Organizations, Assessments, and Information Resources. National Academy Press. Washington, USA.

8. Muhammed PY, Kumar SS (2014) Recent methods for the detection of tumor using computer aided diagnosis - A review.

9. Megha P, Ram M (2011) Aided Diagnosis of Liver Cancer on Computed Tomography Images. Computer Networks and Intelligent Computing, pp. 246-251.

10. Mary G, Albert J, Chi L, Richard E (2014) Direct EM reconstruction of kinetic parameters from list-mode cardiac PET. Nuclear Science Symposium and Medical Imaging Conference (NSS/MIC).

11. Muzi M, O'Sullivan F, Mankoff DA, Doot RK, Pierce LA, et al. (2012) Quantitative assessment of dynamic PET imaging data in cancer imaging. Magn Reson Imaging 30(9): 1203-1215.

12. Ross SG, Gullberg GT, Huesman RH (1997) The effect of heart motion on kinetic parameter estimates for dynamic cardiac SPECT. IEEE Transactions on Nuclear Science 44(3). 
13. Bueno-Orovio A, Britton O, Muszkiewicz A, Rodriguez (2015) Cardiac Modeling. Reference Module in Biomedical Sciences Encyclopedia of Cell Biology 4: 86-94.

14. Narjes Ben Ameur, Nawres Khlifa, Tarek Kraiem (2014) Parametric images for the assessment of cardiac kinetics by magnetic resonance imaging (MRI). Image Processing, Applications and Systems Conference (IPAS), 2014 First International.

15. https://doi.org/10.1371/journal.pone.0118263

16. Bulent S (2007) Proceedings ENTERFACE 2007, Summer Workshop on Multimodal Interfaces. Bogazici University, Turkey.

17. Nelson A, Luciana M (2008) Handbook of Developmental Cognitive Neuroscience. Massachusetts Institute of Technology Press, USA

18. Nidhi, Naveen (2014) A review on Video Quality Assessment. Engineering and Computational Sciences (RAECS).
19. Vijay S, Martin R, Lina J (2011) Objective Video Quality Assessment Methods: A Classification, Review, and Performance Comparison. IEEE Transactions on Broadcasting, 57(2).

20. Tunc O, Martin C, Karol M, Hans P (2010) Video Quality Assessment for Computer Graphics Applications. Max-Planck-Institut Informatik.

21. Beatrice P, Marco C, Frederic D (2016) Motion Estimation Techniques. TELECOM Paris Tech.

22. Motion Estimation Techniques for Digital Video Coding. Metkar S, Talbar S. 2013, XII, 64p. 32 illus. Softcover. ISBN: 978-81-322-1096-2.

23. Heart MRI video with Mill's heart sound 1. Kousakunin, HBSNS.

24. Bulent S (2007) Proceedings ENTERFACE 2007: Summer Workshop on Multimodal Interfaces. Bogazici University, Turkey.

\section{Your next submission with Juniper Publishers} will reach you the below assets

- Quality Editorial service

- Swift Peer Review

- Reprints availability

- E-prints Service

- Manuscript Podcast for convenient understanding

- Global attainment for your research

- Manuscript accessibility in different formats

( Pdf, E-pub, Full Text, Audio)

- Unceasing customer service

Track the below URL for one-step submission

https://juniperpublishers.com/online-submission.php 